\title{
No news from Norway
}

\section{Joshua R. Karas $\cdot$ Roberto Bergamaschi}

Accepted: 8 January 2010 /Published online: 13 February 2010

(C) Springer-Verlag 2010

\section{Dear Editor:}

We read with interest the paper by Hoel et al. (Prolapse of the rectum, long-term results of surgical treatment. Int J Colorectal Dis 2009 Feb;24(2):201-7) and unfortunately have the following semantic, methodological, and editorial concerns:

The awkward use of terms such as "patency of the operation" and "anal insufficiency" is confusing to the reader. Without belaboring the semantics, these are indeed the paper's primary endpoints. Hence, it is appropriate to expect a nomenclature consistent with the international literature. However, we appreciate the authors' optimistic choice to look at the bright side (success rates and durability of surgery) rather than failure.

The authors' finding that recurrence rates following abdominal surgery are lower than recurrence rates after perineal surgery is no news to the reader familiar with the literature. Moreover, patients having undergone abdominal or perineal surgery are not comparable.
The authors may indeed have been unaware of the guidelines regulating the conclusion section of a scientific article, but the reviewers may have known better. The authors did not report their own data on stapled trans-anal rectal resection and thereby provided the reader with unwarranted conclusions. Moreover, the conclusion in the abstract presents a conflicting and unclear message by stating that the perineal approach should be reserved for those with a "very" short life expectancy. Perhaps the authors intended to suggest that a perineal approach should be utilized in patients with a highrisk profile, as we are sure any elective, non-emergent surgical procedure would not be performed on a patient with a short life expectancy. Furthermore, such a statement leaves out the young male patient population group, though small, that is important to recognize. These patients should be considered candidates for a perineal approach, since many colorectal surgeons may strongly feel this would decrease any risk for impotence.

J. R. Karas $\cdot$ R. Bergamaschi $(\bowtie)$

Division of Colon and Rectal Surgery,

State University of New York,

HSC, Level 18, Rm. 046B,

Stony Brook, NY 11794-8191, USA

e-mail: rcmbergamaschi@gmail.com 